\title{
Dorsal Augmentation Using Costal Cartilage: What Is the Best Way?
}

\author{
Yong Ju Jang (iD \\ Department of Otolaryngology-Head and Neck Surgery, Asan Medical Center, University of Ulsan College of Medicine, Seoul, Korea
}

The problematic aspect of dorsal augmentation is that the nasal dorsum is the broadest and the most prominent part of the nose; any minor imperfection is easily discernible by an observer. Furthermore, there is virtually no single ideal graft material and method for the nasal dorsum, free of aesthetic complication. Autologous costal cartilage has been advocated as the best material for dorsal augmentation by many prominent surgeons without strong evidence on literature. However, in the realworld practice, due to the difficulty in harvesting and usage as well as the high propensity to aesthetic complications, costal cartilage could not gain wide popularity as silicone implants, particularly in Asian regions.

Autologous costal cartilage can be used as a dorsal implant in various forms [1]. Traditionally, using it in mono-block carved implant form has been the most common and standard way of dorsal augmentation. If a surgeon is skillful and highly experienced with this approach, this would be an ideal option. This is because one can achieve substantial augmentation with adequate hardness and relatively good viability of the cartilage. However, there are potential problems with this approach. It is not easy to create an implant that has a natural shape and a smooth surface. Besides, complications such as warping, graft movability, and visible contour irregularity continue to be bothering problems [2]. To overcome the inherent disadvantage of solid block costal cartilage for dorsal augmentation, we are witnessing a growing interest in using the costal cartilage in the diced form [3]. Diced cartilage for augmentation is relatively free from the risk of warping and displacement. Another advantage of dicing the cartilage is that surgeons can minimize the amount of cartilage harvested as they can maximize the use of even small remnant fragments of the cartilage after septal reinforcement and tip work, thus not necessitating the harvest of a very long and straight piece of costal cartilage. Although diced cartilage is reported to have good viability and improved chondrocyte survival, the critical weakness of this material is its potential for resorption and difficulty in delivering to the nasal dorsum. In order to ease delivery, dorsal augmentation using diced cartilage is generally accompanied by wrapping with fascia, Surgicel, and Alloderm [3-5]. Another way of using diced cartilage is to make it semi-solid by using fibrin glue [6]. Unlike fascia wrapped diced cartilage, this technique may display increased chance of migration of fragments or visible contour of the cartilage piece, especially through the thin skin. Grafting technique using diced cartilage combined with autologous tissue glue (platelet-rich plasma plus fibrin glue) has also been introduced recently [7]. The use of free diced cartilage without a wrapping material is another option which is suitable for camouflage purpose and minor dorsal augmentation. The final shaping must depend on molding the dorsal contour with fingers and meticulous taping and splinting [8]. Taken together, the trend of dorsal augmentation using costal cartilage is being shifted to using diced cartilage, which calls for a further refinement by technical modification and evidence-based outcome researches.

\section{CONFLICT OF INTEREST}

No potential conflict of interest relevant to this article was reported.

\section{ORCID}

Yong Ju Jang https://orcid.org/0000-0001-7631-0388

\section{REFERENCES}

1. Yoo SH, Jang YJ. Rib cartilage in Asian rhinoplasty: new trends. Curr Opin Otolaryngol Head Neck Surg. 2019 Aug;27(4):261-6.

2. Joo YH, Jang YJ. Comparison of the surgical outcomes of dorsal augmentation using expanded polytetrafluoroethylene or autologous costal cartilage. JAMA Facial Plast Surg. 2016 Sep;18(5):327-32.

Copyright (C) 2019 by Korean Society of Otorhinolaryngology-Head and Neck Surgery.

This is an open-access article distributed under the terms of the Creative Commons Attribution Non-Commercial License (http://creativecommons.org/licenses/by-nc/4.0)

which permits unrestricted non-commercial use, distribution, and reproduction in any medium, provided the original work is properly cited. 
3. Daniel RK. Diced cartilage grafts in rhinoplasty surgery: current techniques and applications. Plast Reconstr Surg. 2008 Dec;122(6): 1883-91.

4. Erol OO.The Turkish delight: a pliable graft for rhinoplasty. Plast Reconstr Surg. 2000 May;105(6):2229-41.

5. Gordon CR, Alghoul M, Goldberg JS, Habal MB, Papay F. Diced cartilage grafts wrapped in AlloDerm for dorsal nasal augmentation. J Craniofac Surg. 2011 Jul;22(4):1196-9.

6. Tasman AJ. Replacement of the nasal dorsum with a diced cartilage glue graft. Facial Plast Surg. 2019 Feb;35(1):53-7.

7. Bullocks JM, Echo A, Guerra G, Stal S, Yuksel E. A novel autologous scaffold for diced-cartilage grafts in dorsal augmentation rhinoplasty. Aesthetic Plast Surg. 2011 Aug;35(4):569-79.

8. Kreutzer C, Hoehne J, Gubisch W, Rezaeian F, Haack S. Free diced cartilage: a new application of diced cartilage grafts in primary and secondary rhinoplasty. Plast Reconstr Surg. 2017 Sep;140(3):461-70.

Received July 26, 2019 Accepted August 5, 2019 